Impacto del contexto municipal sobre el desempeño académico individual

\author{
Manuel Rodríguez y Byron Correa
}


Lecturas de Economía, 90 (enero-junio 2019), pp. 159-193

\section{Manuel Rodríguezy Byron Correa}

\section{Impacto del contexto municipal sobre el desempeño académico individual}

Resumen: Esta investigación tiene como objetivo evaluar la relevancia del contexto municipal sobre el rendimiento académico individual. Para esto, se utilizan microdatos del Instituto Colombiano para la Evaluación de la Educación Icfes e información municipal proveniente del Centro de Estudios sobre Desarrollo Económico. A partir de estos datos se evalúa la incidencia municipal por medio de variables asociadas a la tasa de mortalidad infantil, la violencia y los conflictos de tierras del siglo XX. Lo anterior bajo un escenario multinivel y considerando interacciones entre los distintos contextos. Entre los resultados se destaca la escasa incidencia municipal sobre el rendimiento individual. Asi mismo, se encuentra evidencia en favor de la bipótesis de triple riesgo y una fuerte relación entre el efecto escuela y la composición socioeconómica de la institución educativa.

Palabras clave: educación, municipios, interacción social, nivel socioeconómico, desigualdad.

Clasificación JEL: I21, I24, I25, R10.

\section{The impact of the municipal context on individual academic performance}

Abstract: This research aims to assess the relevance of the municipal context on individual academic performance. To this end, micro data from the Instituto Colombiano para la Evaluación de la Educación Icfes and municipal information from the Centro de Estudios sobre Desarrollo Económico are used. Using these data sources, municipal incidence is evaluated by means of variables associated with the infant mortality rate, violence and land conflicts in the twentieth century, among others. All these analyses are carried out under a multilevel stage and considering interactions between different contexts. Among the results, the limited impact of the municipal context on individual performance stands out. Also, evidence in favour of the triple risk bypothesis is found, as well as a strong relationship between the school effect and the socioeconomic composition of the classroom.

Keywords: education, municipalities, social interaction, socioeconomic level, inequality.

JEL Classification: $I 21, I 24, I 25, R 10$.

\section{L'impact du contexte municipal sur le rendement scolaire individuel}

Résumé: Cette recherche vise à évaluer l'impact du contexte municipal dans le rendement scolaire. Pour cela, on utilise des microdonnées mises à disposition aussi bien par l'Institut Colombien d'Évaluation de l'Éducation-ICFES-que par le Centre d'Études sur le Développement Économique. Sur la base de ces données, l'incidence des facteurs municipaux est évaluée au moyen de variables associées au taux de mortalité infantile, la violence et les conflits fonciers du XXe siècle. Nous considérons un scénario à plusieurs niveaux tout en tenant compte des interactions entre différents contextes. Parmi les résultats, on montre la faible incidence des facteurs municipaux sur le rendement scolaire individuel. De même, nous montrons qu'il existe des preuves en faveur de l'bypothèse du triple risque et d'une relation étroite entre l'effet école et la composition socio-économique de l'institution éducative.

Mots-clés: éducation, municipalités, interaction sociale, niveau socio-économique, inégalité.

Classification JEL: I21, I24, I25, R10. 


\title{
Impacto del contexto municipal sobre el desempeño académico individual $^{*}$
}

\author{
Manuel Rodríguez $\mathbb{1}^{\mathrm{a}}$, Byron Correa $\mathbb{( \mathbb { D }}^{\mathrm{b}}$ \\ -Introducción. -I. Estado del arte. -II. Marco teórico. -III. Metodología y descripción de \\ datos. -IV. Estimaciones y análisis de resultados. -Conclusiones. -Referencias.
}

doi: 10.17533/udea.le.n90a06

Primera versión recibida el 5 de junio de 2018; versión final aceptada el 16 de octubre de 2018

\section{Introducción}

La educación es vista como un motor de desarrollo, en términos de capacidades y libertades (Sen, 1999), así como un medio para mejorar la eficiencia técnica y el crecimiento económico. Desde una mirada más individual, la educación desarrolla las habilidades cognitivas, lo cual incide de forma directa en la productividad de quienes se educan. Por lo tanto, a la luz de la teoría estándar de la firma, aquellos agentes deberían recibir remuneraciones acordes a sus mejoras en la productividad. Desde la sociología se argumenta que la educación es una fuerza con capacidad para cambiar y transformar a una sociedad, pero que al mismo tiempo, por el contrario, puede actuar como una forma de reproducción de disparidades imperantes (Brunner, 2010).

Por estas y otras razones, la educación es considerada como una de las principales herramientas para promover el desarrollo social y económico de

* Una versión preliminar de este documento fue presentada en el XV concurso de ponencias Jesús Antonio Bejarano, en donde fue elegida como la mejor en su categoría.

a Manuel Alejandro Rodríguez Ortiz: estudiante de la Maestría en Economía y asistente de investigación, Departamento de Economía, Escuela de Economía y Finanzas, Universidad Eafit, Medellín, Colombia, Dirección electrónica: marodriguo@eafit.edu.co.

https://orcid.org/0000-0003-0418-4145

b Juan Byron Correa Fonnegra: profesor e investigador, miembro del Grupo de investigación, Economía Regional y Ambiental (GERA), Departamento de Economía, Universidad del Valle, Cali, Colombia. Dirección postal: 760032. Dirección electrónica: Dirección electrónica: juan.correa@correounivalle.edu.co.https://orcid.org/0000-0002-7792-8005 
un país. Así, en América Latina se ha reconocido como un instrumento que apoya simultáneamente varios objetivos políticos, tales como combatir la pobreza y la desigualdad, fomentar la democracia y aportar el desarrollo económico (Duarte, Bos \& Moreno, 2009).

Diferentes investigaciones sobre Colombia dejan ver que el rendimiento de los estudiantes depende, además de las habilidades cognitivas, de las características de la escuela y de sus prácticas académicas (Banco Mundial, 2009). Algunos estudios hacen una fuerte diferencia entre las prácticas e incentivos que coexisten en las escuelas privadas frente a las escuelas públicas (Gaviria \& Barrientos, 2001), pero la mayoría se olvida de medir el grado de interacción social o incluso de incluir escenarios más amplios como el barrio o el municipio donde reside el estudiante. De ahí la relevancia de este estudio, el cual busca examinar las interconexiones e interacciones entre la escuela, el hogar y el municipio donde residen los estudiantes, para entender cómo estos factores repercuten en su rendimiento. Para el caso del contexto escolar, en la literatura nacional se han planteado efectos fijos por escuela (Gaviria \& Barrientos, 2001), pero estos ignoran las posibles interacciones entre el hogar, la escuela y el municipio

Para el caso municipal, la evaluación es pertinente, dada la heterogeneidad y el atraso entre regiones que persisten en el país (Galvis \& Meisel, 2010). Además, se torna interesante si pensamos en variables como la violencia, la calidad de los derechos de propiedad o los indicadores de salud y gestión municipal, cuya influencia no es captada totalmente por el hogar o la escuela y cuya omisión ignora la dinámica del contexto municipal. Dicha dinámica es muy variada para el caso colombiano, pues existen regiones que sufren un cruento conflicto armado, mientras que otras son víctimas de diversas modalidades de hurto, extorsiones y secuestros. También es posible que en otras regiones apartadas no exista violencia, pero sí el clientelismo, la ineficacia y la corrupción, que son la ley imperante. Una vez se enlacen todos los niveles y se estimen los modelos, se podrían responder preguntas como: ¿Existe un efecto municipal sobre la educación en Colombia?, ¿cuál es su magnitud?, $¿$ las interacciones entre contextos son relevantes para explicar el rendimiento educativo?, ¿qué efecto es más preponderante: el escolar o el municipal? 
Las respuestas que se obtengan permitirán describir de mejor manera el proceso educativo en Colombia, dar luces sobre nuevas políticas educativas o cuantificar en términos de desempeño educativo el costo de la guerra, la violencia y demás prejuicios que se escapan de las manos de una administración local.

La estructura del documento es la siguiente: en la primera sección se realizó una introducción que expone la motivación y los objetivos de la investigación; en la sección I se hace una breve revisión de la literatura internacional y nacional, y se examinan algunos de los debates sobre la eficiencia de las escuelas y la influencia municipal; en la sección II se presenta el marco teórico; en la sección III se revisan los datos, sus diversas fuentes, el planteamiento de Manski (1993) y cómo a partir de todos estos surge el modelo estadístico a utilizar; en la sección IV se plantean las estimaciones; finalmente se concluye.

\section{Estado del arte}

Coleman et al. (1966) realizan una de las primeras indagaciones sobre los determinantes del logro académico individual. Estos autores plantean que el principal determinante de este logro son los antecedentes familiares y las características del hogar. Esta conclusión fue polémica en su época, pues, aparentemente, las escuelas, no estaban haciendo nada para mejorar la situación de las personas menos favorecidas y el sistema educativo era entonces un simple reproductor de las desigualdades. Una de las posibles razones por las cuales Coleman et al. obtuvieron esos resultados fue por la simplicidad de su estudio, pues tenía una visión insumo-producto, en la cual solo se incorporaban las características fácilmente observables de la escuela y se ignoraban factores como el clima escolar, la calidad docente, el liderazgo, la composición socioeconómica de la escuela, etc. Adicionalmente, los estudiantes están inmersos en aulas y estas, a su vez, en escuelas; sin embargo, esta estructura jerárquica no fue tenida en cuenta y, como mencionan Raudenbush y Byrk (1986), las estimaciones tradicionales de mínimos cuadrados ordinarios ignoran la dependencia que existe entre los estudiantes de una misma escuela; por tanto, los estimadores resultantes serán ineficientes. 
Posteriormente y mediante un análisis puramente descriptivo, Hanushek (1996) hace un diagnóstico del sistema educativo de Estados Unidos. El autor recalca el crecimiento sostenido y abrupto de los recursos de las escuelas públicas y muestra que los políticos deseaban saber cuánto dinero se debía gastar en educación. Sin embargo, concluye que esa no es la pregunta relevante, sino que sería saber en qué se debía gastar el dinero de la educación, pues en su análisis encuentra que la burocracia educativa y la infraestructura fueron las mayores beneficiadas con los aumentos presupuestales, mientras que el crecimiento salarial de los maestros apenas se evidenció. Los incrementos presupuestales se vieron reflejados en un aumento en los años de educación promedio de la población, aunque surgieron dudas acerca de la calidad de la educación que recibieron los estudiantes. Al revisar los promedios del SAT (Shoclastic Aptitud Test), el autor concluye que, en el mejor de los casos, los resultados se han mantenido constantes y los amplios aumentos presupuestales no se han transformado en mejoras en la calidad.

En otro artículo, Hanushek (1997) revisa cuatrocientas publicaciones que investigan el tema de la eficiencia escolar y, además, considera variables como la razón estudiante-maestro y el gasto por estudiante. Su objetivo es examinar la relación entre esas variables y el desempeño académico. Sin embargo, no encuentra una relación consistente, ya que en diversos casos las variables tienen signos contrarios o no son significativas. Este autor argumenta que el aumento en el gasto per se no debería aumentar el rendimiento, pues deben existir los incentivos para que los estudiantes utilicen los nuevos recursos; así mismo, menciona que la reducción en la razón estudiante-profesor se debe al aumento de las "clases especiales", a las cuales son enviados los estudiantes que requieren educación personalizada por diversos motivos de salud.

Algunos autores como Cunha y Heckman (2007) plantean la teoría del ciclo de formación de habilidades, según la cual los antecedentes familiares determinan las condiciones de la primera etapa de formación, considerada como el mayor determinante en el desarrollo de las etapas posteriores. Los autores argumentan que los esfuerzos por mejorar la educación "tardía" (educación secundaria y superior) poco pueden hacer para corregir las brechas que surgieron en las primeras etapas. Estos argumentos parecen ir en sintonía con el informe de Coleman et al. (1966), pero tiene una sutil diferencia, pues los 
autores también comentan que las intervenciones en educación temprana son un importante mecanismo para cerrar las brechas educativas.

La teoría de la formación del ciclo de habilidades es coherente con los hallazgos de Hanushek (1997), puesto que esta teoría afirma que el paso por el sistema educativo no elimina las brechas que provienen desde la primera infancia, mientras que, como se mencionó anteriormente, Hanushek plantea la necesidad de repensar el gasto educativo debido a que no se evidencia una relación gasto-calidad. Todos estos debates estuvieron en el centro de la investigación educativa, pero en los últimos años se ha empezado a examinar la relevancia del contexto.

Soares y Alves (2013) indagan, simultáneamente, sobre el efecto de las municipalidades y las escuelas sobre el rendimiento académico. Para ello, parten de los desarrollos anteriores, es decir, incluyen variables sociodemográficas del estudiante y otras variables asociadas a la escuela y a su contexto. Utilizan una metodología multinivel con tres niveles, con el objetivo de determinar si existe un efecto municipal y cuantificarlo por medio de los residuales del tercer nivel, con lo cual capturan el ambiente educativo municipal. El ambiente educativo municipal es comparado con los índices de calidad publicados por el gobierno de Brasil. De esta manera, ellos demuestran que aquel índice gubernamental está altamente correlacionado con variables socioeconómicas, por lo cual se favorece a las escuelas y municipios con mejor composición socioeconómica, pues el índice propuesto por los autores es por construcción ortogonal a este tipo de variables y, según su opinión, éste sería un mejor indicador de los efectos a nivel de escuela.

Por otra parte, Blanco (2011) presenta tres críticas a la modelación tradicional de dos niveles. En primer lugar, el autor argumenta que las escuelas no son organismos independientes y que muchos de sus procesos y estructura están determinados por el contexto (barrio, municipio) en el que este inmersa, en contraste con la modelación tradicional que asume independencia. En segundo lugar, y al igual que Manski (1993), piensa que la identificación de los efectos de la interacción social es una tarea titánica que escapa al ceterisparibus, pues permitir una variación aleatoria entre municipios y se espera otorgarle cierta libertad al modelo que posibilite expresar dicha complejidad contex- 
tual. Finalmente, el autor argumenta que la información contextual (barrio, municipio) que se logre incorporar no recogería la totalidad de la dinámica subyacente. A raíz de estas críticas, se plantea un modelo multinivel que asume dependencia entre escuelas de un mismo contexto, con el objetivo de examinar la varianza correspondiente a cada interfaz, una vez descontado el efecto de las variables observables. Lo anterior para recordar la relevancia del entorno y de la interacción entre agentes, ya que el autor encuentra que los niveles de escuela y entorno son responsables casi del $40 \%$ de la variabilidad total.

A modo de resumen, para la determinación del logro educativo las investigaciones internacionales evidencian la importancia tanto de la escuela como del municipio, lo cual se ve reflejado en la existencia de efectos escuela y municipales. No obstante, los factores de dotación inicial del estudiante, como su género, la raza y la educación de los padres, son altamente relevantes. Algunas de las posibles razones que evitaron el estudio temprano de la incidencia del contexto sobre la educación pueden ser la controversia alrededor de la existencia del efecto escuela, la dificultad de recolectar datos de diversas localidades o la creencia de que las escuelas son independientes.

Respecto al ámbito nacional, podemos encontrar el trabajo de Gaviria y Barrientos (2001), el cual ofrece un análisis de la incidencia de los factores individuales sobre el rendimiento. En una primera etapa, estos autores evalúan los factores individuales y algunas variables a nivel de escuela, y en una segunda etapa utilizan las mismas variables a nivel individual, pero empleando efectos fijos por colegio para tener un control más exhaustivo de dicho contexto. En este ejercicio se observa una caída en los coeficientes de las variables individuales después de aplicar los efectos fijos por colegio. Para los autores, esto es una clara evidencia de que la mayor parte del efecto de las variables familiares se potencia en la escuela. En el segundo capítulo de su investigación, Gaviria y Barrientos examinan el efecto diferencial de las características del plantel entre colegios públicos y privados. Ellos encuentran que las características de los colegios tienen mayores impactos en las instituciones privadas; esa diferencia se deriva de un problema de incentivos en los colegios públicos. Pero, si bien ellos mencionan la importancia de la interacción social y del efecto par en la especificación de los modelos, ninguna variable 
recoge explícitamente este efecto. Dado que las instituciones privadas poseen una mejor composición socioeconómica, la variable ficticia asociada al tipo de plantel puede estar absorbiendo el efecto de los pares, aunque estarían asumiendo que este es constante entre estudiantes de un mismo colegio, por lo cual queda un sin sabor con respecto a ese problema de incentivos.

El trabajo de Núñez et al. (2002) plantea que la educación pública es de mala calidad por los terribles incentivos que rodean a los sindicatos de maestros. No obstante, encuentran que las instituciones públicas otorgan mayor rendimiento a los estudiantes de bajos ingresos y que la diferencia entre instituciones públicas y privadas es persistente, a pesar de los diversos controles. Sin embargo, omiten algún tipo de interacción entre el entorno familiar y el contexto escolar.

El logro educativo también es examinado desde una óptica más agregada, pero no necesariamente más amplia. Tanto Mina (2004) como Gärtner (2014) utilizan un panel de datos y como variable dependiente usan el rendimiento promedio del municipio en todas las pruebas. El estudio de Mina (2004) es más amplio, pues considera el efecto de la geografía y demás variables, como índices de pobreza, y encuentra evidencia a favor de la influencia del gasto sobre el rendimiento, particularmente en zonas rurales. Por otro lado, Gärtner (2014) solo se centra en el efecto de la geografía sobre el rendimiento. Ninguno de estos estudios encuentra evidencia de que variables asociadas a la violencia incidan sobre el rendimiento promedio del municipio.

Rozo (2015) evalúa el efecto vecindario sobre el rendimiento. Dicha evaluación la realiza bajo un contexto multinivel, más exactamente considerando tres niveles, y utilizando el formulario de inscripción de la prueba Saber 11, el censo de 2005 y la encuesta C600 del DANE, con lo cual puede construir una base de datos extensa, tanto a nivel individual como de vecindarios y de colegios. Dado el contexto multinivel, también se propone examinar cuál de los dos anidamientos explica más varianza, si el vecindario o el colegio. Entre sus resultados se destaca la existencia del efecto vecindario, pero ese nivel solo explica entre el $4 \%$ y el $8 \%$ de la varianza total para Matemáticas y Lenguaje, respectivamente. 
La literatura nacional ya ha abordado los efectos contextuales en un tercer nivel, del cual se desprende la existencia de un efecto barrio. También se ha examinado la incidencia de la violencia sobre el rendimiento promedio municipal y no se corroboró la existencia de algún efecto sobre el logro educativo. Este estudio trata de unir ambos planteamientos, al considerar un tercer nivel más amplio que el vecindario y los resultados individuales en lugar de promedios municipales, todo esto bajo un escenario de interacción social.

\section{Marco teórico}

La base teórica de la presente investigación se encuentra en Vivas (2008), quien desarrolla un modelo analítico con el objetivo de plasmar los mecanismos y los factores que explican el desempeño escolar. Los factores propuestos son los siguientes: las características del entorno familiar; los atributos de localización (efecto vecindad); los perfiles de oferta educativa (características de la escuela); los hábitos de estudios, el talante y las capacidades individuales innatas; y, finalmente, la esfera gubernamental (políticas educativas y recursos).

Los mecanismos mediante los cuales los factores inciden en el logro académico serían los siguientes.

Las características del entorno familiar tienen al menos dos efectos: el primero es que los padres desean brindar a los hijos un buen capital humano y, el segundo, es que estos deciden la naturaleza de la institución (privada o pública) donde estudiarán sus hijos, así como el tipo de comunidad donde van a vivir. Esta forma de concebir el mecanismo viene del modelo de Benabou (1996).

Los atributos de localización son producto de una decisión de los padres; no obstante, estos afectan el desarrollo y desempeño de los hijos. Conceptos como el capital social y el capital cultural (Bourdieu, 1997) están fuertemente asociados con los atributos de localización: el primero abarca los vínculos sociales de una persona (redes e instituciones) y el segundo se refiere a la cultura que un individuo logra incorporar; una parte de esta cultura puede ser transmitida entre generaciones, pero otra proviene del intercambio, las vivencias y las experiencias. Dependiendo del contexto elegido por 
los padres, sus hijos pueden beneficiarse de estos capitales y potenciar su aprendizaje.

Los perfiles de oferta educativa se refieren a las características de la escuela, como la infraestructura, la composición socioeconómica de los estudiantes, la calidad docente y la eficiencia técnica de la escuela. Dichas variables están asociadas a insumos de la escuela, su proceso y su contexto, las cuales van ligadas al desarrollo del School Effectiveness Research (SER).

Los hábitos de estudio, el talento y las capacidades individuales innatas, aunque en primera instancia pueden parecer independientes de los demás factores, no lo son. Su influencia se potencia o se debilita al entrar en contacto con los demás, especialmente cuando el estudiante interactúa con sus compañeros (efecto de los pares). Aunque las interacciones sociales pueden ser benéficas, también pueden provocar que los estudiantes queden aún más relegados (Willms, 2003).

Finalmente, la esfera gubernamental se refiere a las políticas educativas de orden nacional, departamental o municipal. Adicionalmente, el ámbito institucional posee una alta heterogeneidad en el país, pues parece existir una relación centro-periferia, en donde el centro goza de un cierto grado de desarrollo e inclusión política y social, mientras en la periferia se manifiesta la ley del más fuerte y las instituciones son altamente extractivas (Robinson, 2016). Las elites en el centro deben conceder ciertos derechos y garantías civiles a sus ciudadanos para mantenerse en el poder, pero en la periferia esto no es necesario y el único interés de la elite es preservar el sistema y extraer el máximo beneficio de sus ciudadanos (Acemoglu \& Robinson, 2012). Lo anterior implicaría que, en aquellos municipios con instituciones extractivas, los políticos no tienen entre sus prioridades mejorar la calidad de la educación y, en general, poco les importa el bienestar de sus ciudadanos, por lo que se espera que exista una correlación negativa entre la calidad de las instituciones y el rendimiento académico.

El marco teórico nos indica que, efectivamente, las variables municipales importan, y entre mejores atributos de localización posea un municipio o una localidad mejor será el desempeño de los estudiantes que residen en él. Además, la metodología abordada se basa también en los desarrollos de Manski 
(1993) y Raudenbusch y Bryk (1986). El primero de estos artículos plantea el uso de modelos lineales y la identificación de los distintos efectos que surgen de los procesos de interacción social conceptualizados de la siguiente manera:

- Efectos endógenos: el comportamiento del individuo varía conforme cambia el comportamiento del grupo; en otras palabras, el rendimiento de un estudiante depende del rendimiento medio del curso.

- Efectos contextuales o exógenos: el comportamiento del individuo depende de las características exógenas del grupo; de otra forma, las características exógenas de los compañeros de clase (capital social, riqueza, etc.) ejercen una influencia sobre el rendimiento del estudiante.

- Efectos correlacionados: los individuos y el grupo en el que están inmersos tienden a comportarse de manera similar, pues enfrentan el mismo entorno familiar y/o institucional.

Finalmente, Manski (1993) plantea la siguiente relación:

$$
R=\alpha+\beta E(R \mid G)+E(F \mid G)^{\prime} \gamma+F^{\prime} \lambda+U,
$$

en donde $R$ denota el rendimiento del estudiante; $G$, el grupo o contexto en el que está inmerso; $F$ contiene los factores familiares, y el término $U$ recoge la habilidad no observable. El parámetro $\beta$ capta el efecto endógeno; $\gamma$, los efectos contextuales, y $\lambda$, el efecto directo de las características observables del estudiante.

Pero el autor supone que al controlar por $F$ y $G$, entonces

$$
E(U \mid G, F)=G^{\prime} \delta,
$$

donde $\delta$ captura el impacto de los efectos correlacionados.

Después de resolver el modelo, el autor describe el equilibrio social. Es ahí en donde se evidencia el papel de los efectos endógenos como multiplicador social, pues si un estudiante mejora su rendimiento, el rendimiento del grupo en el que está inmerso también aumentará, y ese aumento endógenamente reforzará el incremento inicial en el rendimiento. Los otros dos efectos no poseen esta característica. 
Por otro lado, Raudenbusch y Bryk (1986) plantean los problemas metodológicos que pueden surgir de la omisión de estructuras multinivel, es decir, de no tener en cuenta el anidamiento de los datos, hecho que fue bastante común en los estudios de educación hasta hace algunos años. La principal falencia de no trabajar con una estructura multinivel es el aumento en la probabilidad de cometer un error tipo I, lo cual lleva a aceptar efectos causales que son espurios. Aunque ambos autores tocan el tema de las interacciones sociales, sus planteamientos metodológicos no están muy ligados. Allí aparece nuevamente Vivas (2008), quien parte de la ecuación de equilibrio social de Manski (1993):

$$
E(R \mid G, F)=\frac{\alpha}{1-\beta}+E(F \mid G)^{\prime}\left[\frac{\gamma+\lambda \beta}{1-\beta}\right]+G^{\prime}\left[\frac{\delta}{1-\beta}\right]+F^{\prime} \lambda
$$

Dicha ecuación muestra el rendimiento esperado de un estudiante condicionado a sus atributos $(F)$ y a las características del grupo en el que está inmerso $(G)$. Se observa que la identificación de los efectos endógenos, correlacionados y contextuales es difícil, puesto que el impacto de cada variable es una combinación de los diferentes efectos.

No obstante, la estrategia de identificación se basa en descomponer el efecto correlacionado en dos partes: una primera parte asociada a las variables observables del colegio y una segunda parte totalmente aleatoria:

$$
G^{\prime}\left[\frac{\delta}{1-\beta}\right]=\rho C+E,
$$

donde $E$ es un vector que contiene los efectos aleatorios asociado a cada uno de los $j$ colegios, mientras $C$ puede ser un índice asociado al clima escuela o cualquier conjunto de variables observables a nivel de colegio. Por lo tanto, el rendimiento de un estudiante $i$ en una escuela $j$ será el siguiente:

$$
R_{i j}=\alpha^{*}+\rho C_{j}+\theta F_{(-i) j}^{*}+\lambda F_{i j}+e_{j}+u_{i j},
$$

en donde

$$
\frac{\alpha}{1-\beta}=\alpha^{*}
$$


Rodríguez y Correa: Impacto del contexto municipal sobre el desempeño académico individual

$$
E(F \mid G)^{\prime}\left[\frac{\gamma+\lambda \beta}{1-\beta}\right]=\theta F^{*} .
$$

Mediante esta especificación se puede identificar directamente el impacto de los atributos propios del estudiante y del colegio; además, se puede recopilar información valiosa sobre los demás efectos.

A pesar de que el modelo planteado por Vivas (2008) solo contempla dos niveles, podemos plantear la siguiente descomposición:

$$
G^{\prime}\left[\frac{\delta}{1-\beta}\right]=\rho_{1} C+\rho_{2} M+E+\xi,
$$

en donde $M$ es un vector de variables observables a nivel de municipio y $\xi$ es un vector cuyos componentes están asociados a cada municipio; así, se justifica la estructura multinivel que deseamos. Adicionalmente, $F^{*}$ debería incorporar las variables que capturen los atributos comunes de estudiantes que compartan o interactúen algún nivel (como índices socioeconómicos en distintos niveles de agregación).

De esta forma, identificaríamos directamente los parámetros asociados a los atributos propios y obtendríamos el efecto asociado a cada grupo (escuela y municipio), aunque esto debe interpretarse con cuidado. Finalmente, lograríamos alguna idea de la magnitud de la interacción social vía $\theta$ que, si bien inicialmente fue considerado como un escalar, representa un vector que captura los distintos efectos de la interacción social a través de las distintas variables que dependen de la composición de cada nivel (municipio y escuela).

De aquí surge la estructura multinivel que une ambos planteamientos y que será utilizada en el estudio propuesto. En dicha estructura, el rendimiento del estudiante $i$ que asiste al colegio $j$ y está inmerso en el municipio $m$ depende de sus antecedentes familiares $(F)$, de las características de ambos niveles $(G)$ y de la interacción de ambos conjuntos de variables $\left(F^{*}\right)$. Por tanto, el modelo estadístico sería el siguiente:

$$
R_{i j m}=\alpha^{*}+\rho_{1} C_{j m}+\rho_{2} \operatorname{Mun}_{m}+\theta F_{(-i) j m}^{*}+\lambda F_{i j m}+v_{m}+e_{j m}+u_{i j m} .
$$

Ahora la técnica nos permite tener parámetros que varían entre escuelas y, adicionalmente, nos da la capacidad de descomponer la varianza en el número 
de niveles que incorporemos, lo que nos permite una descripción aún más rica del fenómeno estudiado.

$$
\operatorname{Var}\left(R_{i j m}\right)=\operatorname{Var}\left(v_{m}+e_{j m}+u_{i j m}\right),
$$

en el caso de que todos los errores sean independientes, aunque es posible trabajar sin ese supuesto.

$$
\sigma_{i j m}^{2}=\sigma_{m}^{2}+\sigma_{j m}^{2}+\sigma_{i j m}^{2} .
$$

Lo anterior nos permite determinar el origen de la variabilidad en los rendimientos, lo cual es útil para describir de una mejor manera el fenómeno como para focalizar políticas de intervención en un determinado nivel.

\section{Metodología y descripción de los datos}

De las derivaciones teóricas anteriormente descritas, surge la ecuación (9), la cual no es más que una forma reducida del modelo estructural de interacción social de Manski (1993) y de Vivas (2008), donde se considera una estructura jerárquica que da lugar a un modelo multinivel. En esta sección se describe brevemente la estructura general de los modelos multinivel y los supuestos que permiten su estimación.

La estructura básica de este tipo de modelos se expone a continuación. En primera instancia, es necesario tener en cuenta que los individuos (estudiantes) están agrupados en ciertas unidades (escuelas). Consideremos el siguiente modelo:

$$
y_{i j}=\alpha_{j}+\beta_{j} x_{i j}+\varepsilon_{i j}
$$

el cual relaciona una variable $y$ (el rendimiento en la prueba de Matemáticas) con una variable $x$ (el nivel socioeconómico) y una perturbación. Además, el intercepto y la pendiente del modelo son específicos a cada unidad; esta es una de las características del modelo que permite que los coeficientes varíen entre unidades de agregación, lo cual se refleja en las siguientes relaciones:

$$
\begin{aligned}
& \alpha_{j}=\alpha_{0}+u_{j}, \\
& \beta_{j}=\beta_{0}+u_{1 j} .
\end{aligned}
$$


$\mathrm{Al}$ introducir estas relaciones de segundo nivel en la ecuación (12), se obtiene el modelo descrito por la ecuación (15), el cual está compuesto por una parte fija y una parte aleatoria:

$$
\begin{gathered}
y_{i j}=\alpha_{0}+\beta_{0} x_{i j}+u_{1 j} x_{i j}+u_{j}+\varepsilon_{i j}, \\
u_{j} \sim N\left(0, \sigma_{u}^{2}\right), \quad \varepsilon_{i j} \sim N\left(0, \sigma_{\varepsilon}^{2}\right), \quad u_{1 j} \sim N\left(0, \sigma_{u_{1}}^{2}\right) .
\end{gathered}
$$

Este modelo permite que el intercepto y la pendiente sean específicos para cada grupo. En donde $u_{j}$ representa la desviación de la unidad $j$ respecto al promedio general, y $u_{1 j}$ representa el efecto diferencial de la variable $x_{i j}$ en el grupo $j$. En el contexto del estudio, los términos $u_{j}$ y $u_{1 j}$ son variables aleatorias que se consideran como componentes del efecto escuela.

Nótese que, a diferencia de los modelos lineales tradicionales, en este caso se cuenta con tres términos de error. Una forma de estimar los parámetros $\alpha_{0}$ y $\beta_{0}$ es redefinir un error compuesto y posteriormente estimar aquel modelo mediante máxima verosimilitud; después, realizar una descomposición de la varianza para generar la distribución de cada error y así identificarlos.

Entre los supuestos del modelo se considera la independencia entre los términos de error del primer y segundo nivel. Para utilizar el método de máxima verosimilitud se asume que estos siguen una distribución normal, aunque es factible considerar estructuras más flexibles que permitan correlaciones entre los términos de perturbación de los diferentes niveles o en donde aquellos términos no sigan una distribución normal o, incluso, permitir que el modelo no sea lineal. Para una mejor descripción del alcance y poder de los modelos multinivel o en general de los modelos de coeficientes aleatorios, véanse StataCorp (2013) y Goldstein (2011).

\section{A. Datos municipales y exploración espacial}

Los datos utilizados provienen del Panel Municipal del Centro de Estudios de Desarrollo Económico (CEDE) de la Universidad de los Andes y de la prueba Saber 11, ambas fuentes corresponden al año 2013. Una de las motivaciones para incluir el contexto municipal son los contrastes del país, pues al mapear variables asociadas a la calidad institucional, se evidencia una 
relación centro-periferia, en la cual Bogotá y la zona andina disfrutan de una mejor calidad institucional, mientras que en la periferia se observan altas tasas de mortalidad infantil (TMI) y de homicidios, así como poca eficiencia fiscal, como se evidencia en el Gráfico 1.

\section{Gráfico 1. Relación centro-periferia}
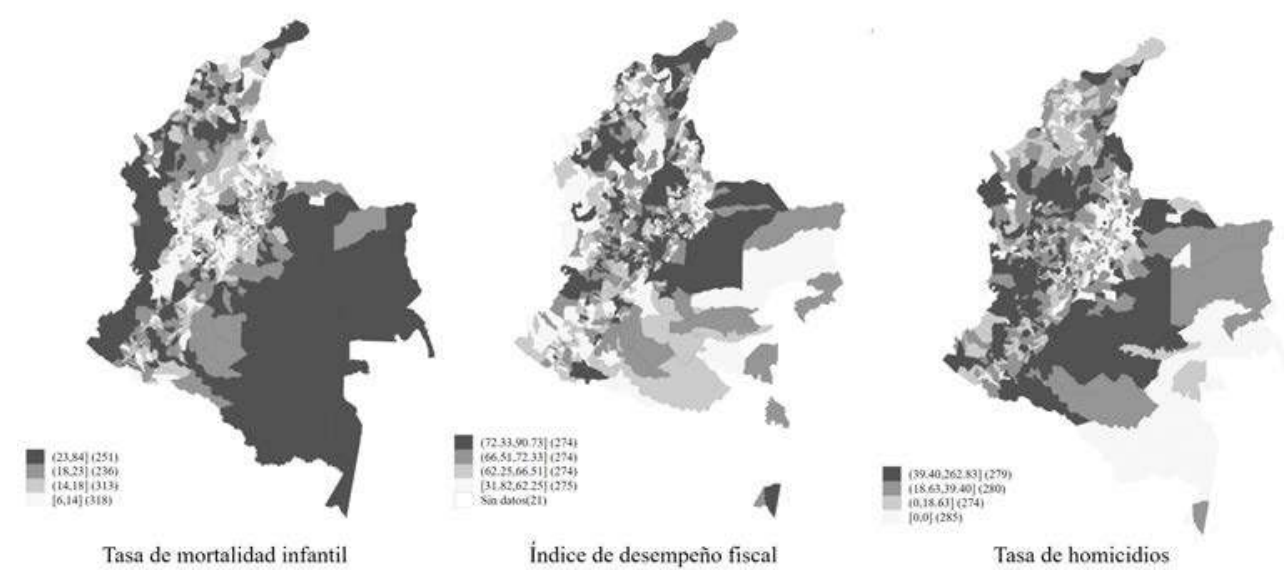

Nota: los colores oscuros denotan cuartiles más altos de las variables, mientras los colores claros, los más bajos.

Fuente: elaboración propia con datos del CEDE.

Esta relación coincide con los clústeres de pobreza encontrados por Galvis y Meisel (2010) y con la relación estudiada por Robinson (2016). Además, la periferia del país también se caracteriza por una escasa infraestructura y una geografía agreste; este tipo de variables fueron significativas en los estudios de Mina (2004) y Gärtner (2014). El hecho de que en la periferia sean incapaces de controlar la TMI, siendo este uno de los indicadores básicos del desarrollo, es una prueba de la negligencia de las instituciones de aquellas regiones. Es posible que esta negligencia se extienda también a la política educativa.

Los conflictos considerados en este estudio se centran en tres periodos de violencia que se desarrollaron durante el siglo pasado en nuestro país: dos conflictos de tierras durante la primera mitad del siglo y el periodo denominado la Violencia, desencadenada por el asesinato de Jorge Eliécer Gaitán. 
Los conflictos de tierras fueron un claro ejemplo de la debilidad institucional del país, pues en una primera instancia los grandes hacendados se apropiaron de extensiones considerables de tierras, pero posteriormente el gobierno creó diversos mecanismos legales para evitar una mayor concentración de la esta; de dichas medidas se beneficiaron muchos colonos, quienes lograron conseguir terrenos. Sin embargo, después los hacendados, volvieron a hacerse con el control sobre los baldíos, ya fuera por la fuerza, la omisión de los decretos nacionales o el engaño a los colonos. Fue entonces cuando varios grupos de colonos desarrollaron una serie de batallas, legales y campales, contra los hacendados por los terrenos baldíos que rodeaban la frontera habitable del país. La mayor parte de estos conflictos ocurrió en las zonas cafeteras de clima medio de las tres cordilleras, las zonas ganaderas del interior y el enclave bananero de la United Fruit Company (Sánchez \& Peñaranda, 1991).

La debilidad institucional, que se manifestó en la incapacidad de darle una solución eficaz y duradera a aquellos conflictos en esas regiones, fue un reflejo de las instituciones extractivas que existían en aquellas zonas en ese entonces. Si efectivamente el sistema extractivo se mantuvo a través de los años, el efecto de vivir en alguno de esos municipios sería negativo. En la Tabla 1 se muestra la cantidad de estudiantes que vivían en lugares que sufrieron conflictos, en el panel A se muestra para los conflictos de tierra y en el panel B para el periodo de violencia.

Tabla 1. Estadisticas de los conflictos en Colombia

\begin{tabular}{cccc|ccc}
\hline \multicolumn{4}{c|}{ Panel A } & \multicolumn{3}{c}{ Panel B } \\
\cline { 1 - 3 } Conflictos & \multicolumn{2}{c|}{$1901-1917$} & & Violencia & Estudiantes & $\%$ \\
\cline { 2 - 4 } $1918-1931$ & No & Sí & Total & $1948-1953$ & & \\
\hline No & 280.852 & 25.291 & 306.143 & No & 376.791 & 92,7 \\
Sí & 58.016 & 42.192 & 100.208 & Sí & 29.56 & 7,3 \\
Total & 338.868 & 67.483 & 406.351 & Total & 406.351 & 100 \\
\hline
\end{tabular}

Fuente: elaboración propia con datos del Icfes y el CEDE. 
Durante el año 2013, aproximadamente el $30 \%$ de los estudiantes que presentaron la prueba Saber 11 residían en municipios en donde se desarrollaron conflictos de tierras, mientras que el $7 \%$ vivía en zonas que fueron afectadas por la Violencia. Aunque estas estadísticas representan el número de estudiantes que para dicho año vivían en estos municipios, es importante reconocer aquellos municipios que se vieron afectados por estos flagelos, puesto que los conflictos que inicialmente se localizaban en la frontera habitable del país se fueron acercando hacia el interior de este, así se evidencia en el Gráfico 2.

\section{Gráfico 2. Distribución espacial de los conflictos}

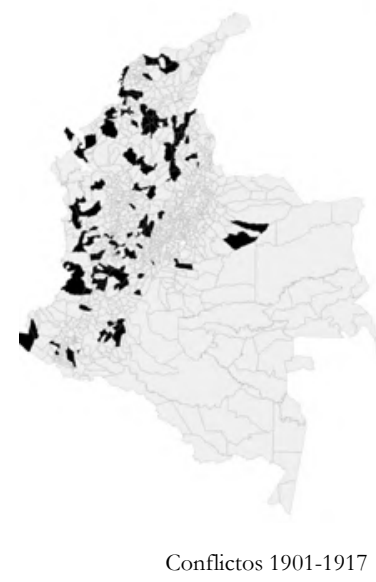

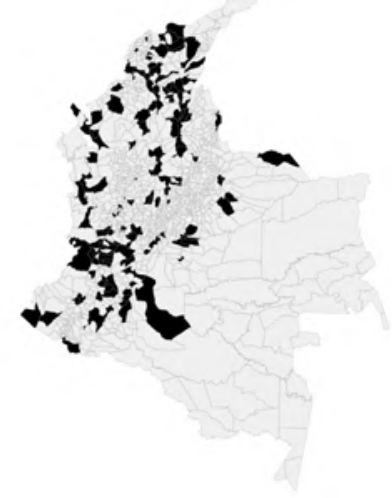

Conflictos 1918-1931

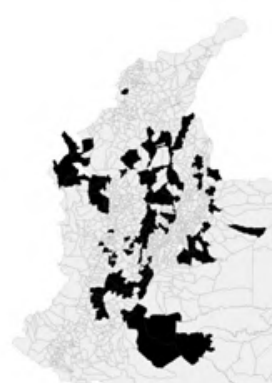

Violencia 1948-1953

Nota: el color negro denota la ocurrencia de conflicto en ciertos municipios.

Fuente: elaboración propia con datos del CEDE.

Cabe mencionar que la fuente de la cual proviene la información sobre los conflictos de tierras es el Archivo Nacional de Colombia y del trabajo realizado por Catherine Legrand, publicado en Sánchez y Peñaranda (1991), mientras que la información correspondiente al periodo de la Violencia proviene de los informes de la Policía Nacional de Colombia recopilados por el CEDE (véase la Tabla 2). 
Rodríguez y Correa: Impacto del contexto municipal sobre el desempeño académico individual

Tabla 2. Estadisticas descriptivas de los datos del CEDE

\begin{tabular}{lccccc}
\hline Variable & N & Media & Desviación & Mín. & Máx. \\
\hline TMI & 1.105 & 19,794 & 8,040 & 6,507 & 82,773 \\
Desempeño fiscal & 1.095 & 67,261 & 7,780 & 31,82 & 90,73 \\
Conflicto - 1901-1917 & 1.105 & 0,091 & 0,288 & 0 & 1 \\
Conflicto - 1918-1931 & 1.105 & 0,122 & 0,327 & 0 & 1 \\
Violencia 1948-1953 & 1.105 & 0,134 & 0,341 & 0 & 1 \\
Tasa de homicidios & 1.105 & 28,395 & 33,096 & 0 & 256.410 \\
\hline
\end{tabular}

Nota: el tamaño muestral representa el número de municipios para los cuales se tiene información.

Fuente: elaboración propia utilizando datos del CEDE.

\section{B. Datos individuales e institucionales}

Los datos recopilados provienen del formulario de inscripción de las pruebas Saber 11 del año 2013. Como medida del desempeño o del logro académico se utiliza el puntaje en la prueba de Matemáticas. Adicionalmente, se consideran los estudiantes cuyas jornadas académicas son en la mañana o jornada completa y se descartan los de las jornadas nocturnas, sabatinas y las que son exclusivamente en la tarde. Las jornadas excluidas representan el $30 \%$ de los datos.

Debido a la importancia de las características familiares sobre el desempeño y desarrollo de los estudiantes, la literatura plantea constructos teóricos abstractos, como el nivel socioeconómico, para expresar la misma idea, pero en función de un menor número de variables. En la práctica, esta estrategia conlleva beneficios, pues evita la inclusión de variables altamente correlacionadas y así se previenen problemas de multicolinealidad; por otro lado, permite realizar interesantes evaluaciones sobre aquellos constructos teóricos.

Para la elaboración del índice del nivel socioeconómico se siguen las recomendaciones del Icfes (2010). Por tanto, es necesario construir, mediante el método de componentes principales, un índice de dotación de los bienes y servicios que posee el hogar. Una vez se tiene este índice, se agregan las variables relacionadas con la educación, la posición social y los ingresos del hogar 
para conjugar mediante el mismo método de componentes principales el índice socioeconómico del estudiante (INSE). A partir de esta variable es posible construir el nivel socioeconómico promedio de la escuela (INSEC), el cual es importante para examinar la influencia de la heterogeneidad contextual sobre el rendimiento. Adicionalmente, Willms (2003) considera la interacción entre los índices socioeconómicos del estudiante y el de su escuela (INSEX) como un indicador importante de la desigualdad de oportunidades en una sociedad.

Supongamos que dicha interacción es significativa y que su signo es positivo. En este caso, se verificaría la hipótesis de triple riesgo, en la cual los individuos con mejores condiciones sociales (INSE alto) y en contextos altamente favorables (INSEC altos) obtienen mejores resultados, no solo por sus propias condiciones o las del contexto sino también por la interacción de ambos. De esta manera, se aumentaría aún más su ventaja sobre las personas desfavorecidas, las cuales no recibirían un triple beneficio, sino que, más bien, sería un triple castigo, dadas sus precarias condiciones sociales y la segregación que los obliga a estar en ambientes desfavorables.

Finalmente, se considera el género del estudiante, puesto que existen distintas teorías y evidencias, tanto a nivel nacional como internacional, de la existencia de una brecha de género (Icfes, 2013). Con el fin de obtener estimaciones más claras de los distintos contextos, se controla por esta variable. En la Tabla 3 se presenta las estadísticas descriptivas de las variables individuales e institucionales.

Tabla 3. Estadísticas descriptivas de los datos del Icfes

\begin{tabular}{lccccc}
\hline Variable & $\mathrm{N}$ & Media & Desviación & Mín. & Máx. \\
\hline Puntaje & 406.351 & 46,097 & 11,072 & 0,000 & 127,00 \\
Género & 406.318 & 0,457 & 0,498 & 0,000 & 1,000 \\
INSE & 406.351 & 0,213 & 2,142 & $-3,836$ & 7,810 \\
INSEX & 406.351 & 3,402 & 6,004 & $-8,953$ & 54,512 \\
INSEC & 8.533 & $-0,058$ & 2,010 & $-3,566$ & 7,278 \\
\hline
\end{tabular}

Fuente: elaboración propia con datos obtenidos del formulario de inscripción del Icfes. 
Rodríguez y Correa: Impacto del contexto municipal sobre el desempeño académico individual

En el Gráfico 3 se presenta la distribución del índice socioeconómico para cada tipo de plantel. Se observa que en los colegios públicos priman los contextos desfavorables y es un reducido número de estudiantes los que tienen condiciones favorables. Por el contrario, en las instituciones privadas son pocos los estudiantes que viven en condiciones desfavorables y muchos los que viven en condiciones adecuadas para recibir educación.

\section{Gráfico 3. Distribución del INSE según el tipo de plantel}

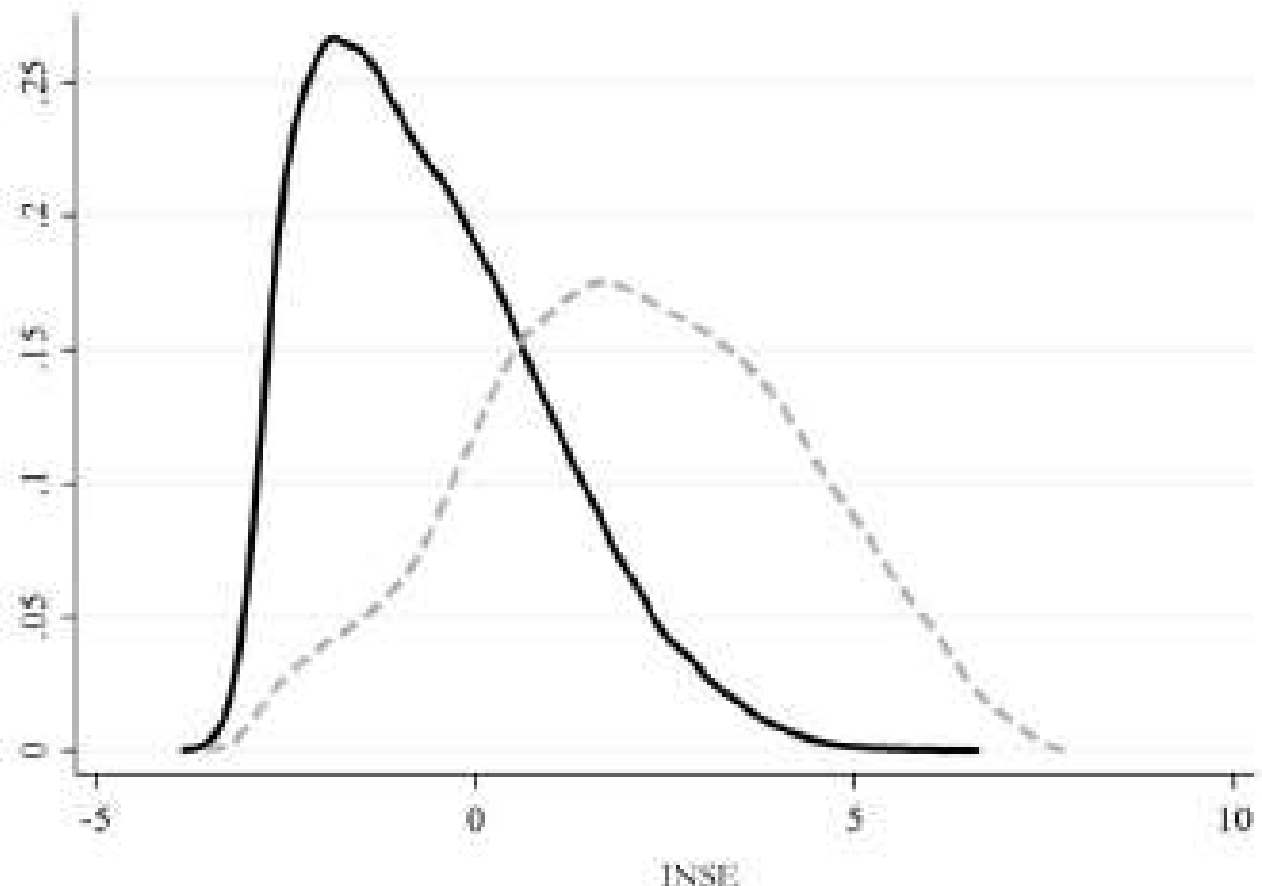

\section{OFICIAL \# NO OFICIAI}

Fuente: elaboración propia con datos del CEDE. 


\section{Relevancia contextual: el modelo nulo}

Como se formuló anteriormente, el rendimiento educativo individual depende de un gran número de factores, de las interacciones entre estos y del anidamiento implícito del estudiante; situación descrita en la ecuación (9). No obstante, antes de aplicar aquel modelo, es necesario examinar la relevancia de una estructura en tres niveles. Por lo tanto, es fundamental estimar el siguiente modelo:

$$
R_{i j m}=\beta_{0}+v_{m}+e_{j m}+u_{i j m},
$$

del cual se obtienen $v_{m}$ y $e_{j m}$, que representan los efectos municipales y escolares, respectivamente. Esta primera aproximación nos permitirá imponer un límite superior para los efectos de cada contexto y, posteriormente, conocer cuál de estos incide en mayor proporción sobre el rendimiento. Al realizar la descomposición de la varianza en cada uno de los niveles, se encuentra que el $29,8 \%$ de la varianza en la prueba de Matemáticas se debe a diferencias entre las escuelas, mientras que el $4,7 \%$ se debe a heterogeneidades municipales. En el Gráfico 4 se presenta la magnitud de los efectos en cada contexto.

En el Gráfico 4 se muestra el efecto asociado a cada unidad contextual y sus correspondientes errores estándar. Se observa que la magnitud de los efectos municipales es considerablemente menor a la que alcanza el efecto colegio, y que una gran parte de los efectos a nivel municipal no son significativos. Sin embargo, aún es posible considerar que el municipio tenga un efecto indirecto, por medio de algunas de las variables a nivel del colegio y del hogar. Por esta razón, es necesario considerar la inclusión de variables y sus interacciones; no obstante, no es imprescindible plantear una estructura de tres niveles.

Por otra parte, es posible examinar el efecto del contexto escolar entre instituciones públicas y privadas. Se encuentra una diferencia significativa de 4 puntos a favor de las instituciones privadas; sin embargo, el subgrupo de las escuelas públicas, a pesar de contar con más de 200.000 estudiantes distribuidos en aproximadamente 5.000 colegios, presenta un rendimiento más homogéneo. Al examinar el mapa de dispersión entre el efecto escuela y el INSEC para cada tipo de institución (Gráfico 5), se manifiesta una correlación positiva entre este efecto y el nivel socioeconómico del colegio. Además, 
Rodríguez y Correa: Impacto del contexto municipal sobre el desempeño académico individual

la relación en los planteles privados parece ser exponencial, situación que no ocurre en las instituciones públicas.

\section{Gráfico 4. Efectos contextuales por grupo}
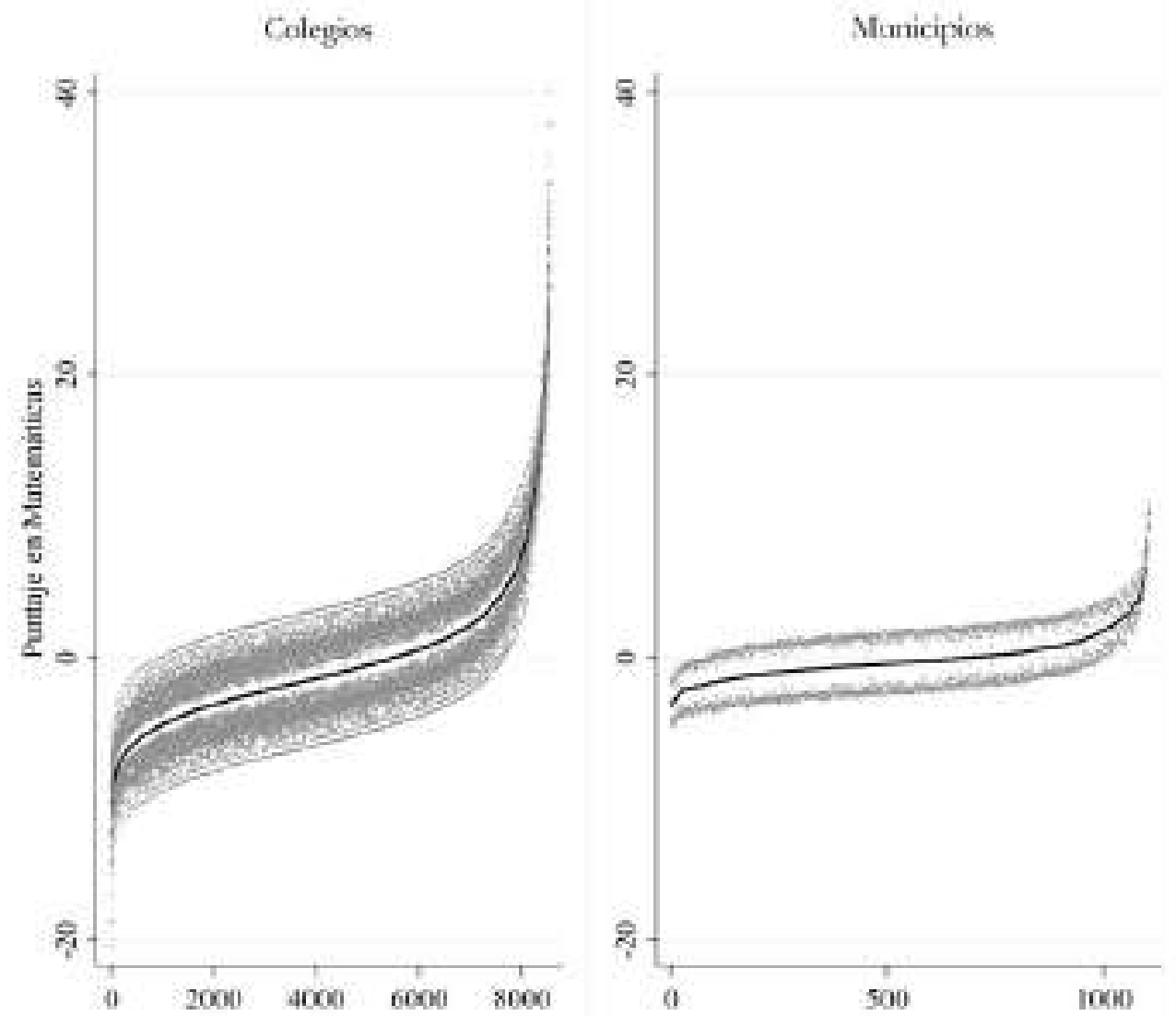

Nota: las líneas negras denotan el efecto de cada grupo, ya sea colegio o municipio. Los puntos grises corresponden a los intervalos de confianza.

Fuente: elaboración propia con datos del Icfes.

Al observar el Gráfico 5, se aprecia que el efecto escuela es más importante en los planteles privados, tanto por número de instituciones con efectos significativos como por magnitud. Mientras tanto, en los planteles públicos son pocas las instituciones con efectos significativos, y en cuanto a la magni- 
tud, solo un puñado otorga 20 puntos. También es evidente que la dispersión entre los planteles es más alta en las instituciones privadas, los índices de correlación intraclase son de $12 \%$ y $37 \%$ para las escuelas públicas y privadas, respectivamente; es decir, el $37 \%$ de la variabilidad de los resultados a nivel individual en los planteles privados proviene de diferencias atribuibles al contexto escolar.

\section{Gráfico 5. Efecto colegio según el tipo de plantel}

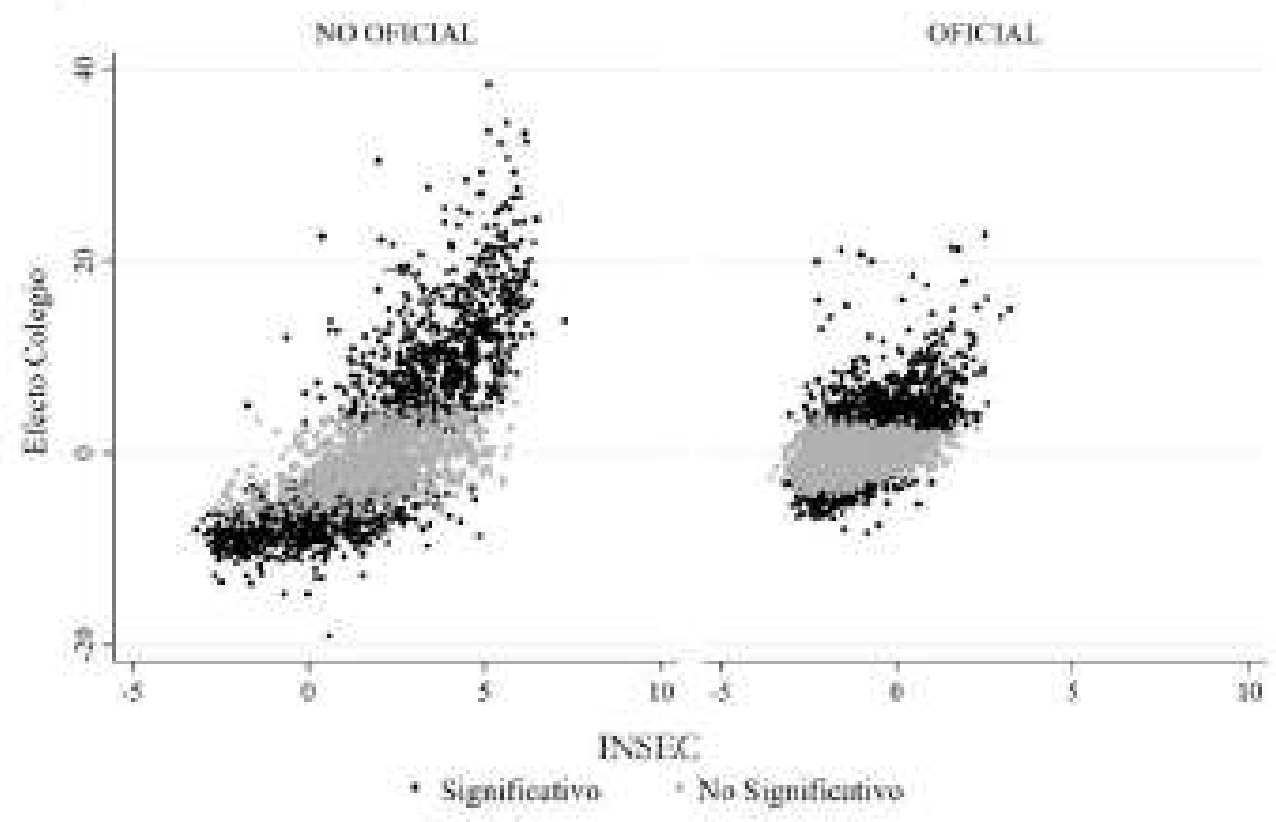

Fuente: elaboración propia con datos del Icfes.

Como se ha evidenciado, el efecto escuela es más preponderante en las instituciones privadas y está altamente correlacionado con el INSEC. ¿Por qué ocurre esto? En primer lugar, es necesario una exploración del nivel socioeconómico del colegio, que desagregue por el tipo de institución.

En la Tabla 4 se muestran las estadísticas descriptivas del INSEC. Allí se observa que los colegios privados tienen estudiantes y entornos en condiciones adecuadas para asistir a la escuela, pues, en promedio, los estudiantes de 
esos planteles asisten bajo mejores condiciones y con un mayor bagaje cultural y social, derivado de su mayor nivel socioeconómico, en comparación con sus pares de los planteles públicos. Ese entorno favorable que surge de la composición del colegio debe estar asociado a mejores resultados académicos a nivel de escuela, de ahí la relación positiva entre el INSEC y el efecto escuela.

Tabla 4. Distribución del INSEC según el tipo de plantel

\begin{tabular}{lccccc}
\hline INSEC & Número de colegios & Media & Desviación & Mínimo & Máximo \\
\hline Oficial & 5.498 & $-1,174$ & 1,902 & $-3,566$ & 3,205 \\
No oficial & 3.035 & 1,852 & 1,010 & $-3,203$ & 7,279 \\
\hline
\end{tabular}

Fuente: elaboración propia con datos del Icfes.

\section{Estimaciones y análisis de resultados}

En esta sección se pretende explicar el efecto escuela y, de manera simultánea, examinar el efecto de diversas variables proxy que miden la calidad institucional a nivel municipal sobre el rendimiento académico. La Tabla 5 muestra los resultados de los 8 modelos considerados bajo una estructura de dos niveles, en la cual los estudiantes están anidados en escuelas. Los coeficientes de las variables que resultan significativas en cada modelo reflejan la influencia de la variable en la explicación del desempeño académico. En el primer modelo solo se considera la influencia de las condiciones del hogar y por tanto únicamente se incluyen variables asociadas al estudiante y a sus padres. La varianza asociada a las escuelas alcanza aproximadamente el $20 \%$ en este caso.

En el segundo modelo se considera la incidencia del tipo de plantel (público o privado) y se evidencia que en promedio los planteles públicos alcanzan puntajes inferiores. Sin embargo, al considerar la interacción entre INSEC y el INSE, este cambia de signo, por lo que los planteles públicos obtienen un rendimiento superior frente a sus homólogos privados. Este resultado es similar al de Orejuela (2014), quien capta el hecho de que únicamente en la parte superior de los colegios privados, estos son mejores a los públicos; esto es, 
solo un puñado de colegios privados se encuentran en esa exclusiva posición. Adicionalmente, la hipótesis de triple riesgo de Willms (2003) es verificada.

Tabla 5. Resultados de los modelos a nivel de escuela

\begin{tabular}{|c|c|c|c|c|c|}
\hline Modelo & 1 & 2 & 3 & 4 & 5 \\
\hline \multirow[t]{2}{*}{ Género } & $3,157^{* * *}$ & $3,161^{* * *}$ & $3,196^{* * *}$ & $3,187^{* * *}$ & $3,184^{* * *}$ \\
\hline & $(0,0304)$ & $(0,0304)$ & $(0,0303)$ & $(0,0303)$ & $(0,0303)$ \\
\hline \multirow[t]{2}{*}{ INSE } & $1,202^{* * *}$ & $1,120^{* * *}$ & $0,856^{* * *}$ & $0,562^{* * *}$ & $0,622^{* * *}$ \\
\hline & $(0,0116)$ & $(0,0122)$ & $(0,0132)$ & $(0,0302)$ & $(0,0309)$ \\
\hline \multirow[t]{2}{*}{ INSEC } & & & $1,738^{* * *}$ & $2,173^{* * *}$ & $1,995^{* * *}$ \\
\hline & & & $(0,0344)$ & $(0,0472)$ & $(0,0524)$ \\
\hline \multirow[t]{2}{*}{ INSEX } & & & $0,124^{* * *}$ & $0,146^{* * *}$ & $0,119^{* * *}$ \\
\hline & & & $(0,00604)$ & $(0,00867)$ & $(0,0092)$ \\
\hline \multirow[t]{2}{*}{ Tipo } & & $-2,683^{* * *}$ & $2,288^{* * *}$ & $2,313^{* * *}$ & $2,170^{* * *}$ \\
\hline & & $(0,114)$ & $(0,128)$ & $(0,127)$ & $(0,109)$ \\
\hline \multirow[t]{2}{*}{ Tipo-INSE } & & & & $0,403^{* * *}$ & $0,336^{* * *}$ \\
\hline & & & & $(0,0352)$ & $(0,036)$ \\
\hline \multirow[t]{2}{*}{ Tipo-INSEC } & & & & $-0,900^{* * *}$ & $-0,783^{* * *}$ \\
\hline & & & & $(0,073)$ & $(0,0722)$ \\
\hline \multirow[t]{2}{*}{ Constante } & $40,76^{* * *}$ & $45,19^{* * *}$ & $36,48^{* * *}$ & $36,03^{* * *}$ & $36,29^{* * *}$ \\
\hline & $(0,069)$ & $(0,2)$ & $(0,222)$ & $(0,225)$ & $(0,199)$ \\
\hline$N$ & 406.265 & 406.265 & 406.265 & 406.265 & 406.265 \\
\hline ICC & 0,195 & 0,188 & 0,130 & 0,128 & 0,067 \\
\hline
\end{tabular}

Nota: errores estándar entre paréntesis. ${ }^{*} \mathrm{p}<0,1,{ }^{* *} \mathrm{p}<0,05,{ }^{* * *} \mathrm{p}<0,01$

Fuente: elaboración propia con datos del Icfes.

En el cuarto modelo se consideran las interacciones con el tipo de plantel. Se observa que en las instituciones públicas se potencian los factores del hogar, mientras que, en los colegios privados, el INSEC es la variable más preponderante. En este punto la varianza no explicada asociada a los colegios alcanza aproximadamente el 13\%. En el quinto modelo se consideran efectos aleatorios asociados al INSEC, para permitir que el efecto de esta variable 
Rodríguez y Correa: Impacto del contexto municipal sobre el desempeño académico individual

cambie entre instituciones; esta estrategia reduce el índice de correlación intraclase al $7 \%$. El modelo completo a nivel de escuela será el quinto, el cual considera factores individuales, la composición socioeconómica del curso, los efectos aleatorios a nivel de plantel y de INSEC, así como las interacciones entre distintas variables.

En cuanto a la interacción de los contextos a nivel de hogar y de colegio, se valida la hipótesis de triple riesgo de Willms (2003), quien plantea que el sistema educativo colombiano es un reproductor de desigualdades, en el cual los estudiantes menos aventajados socieconómicamente sufren 3 tipos de penalizaciones: en primer lugar, por su desventaja inicial, luego por su contexto desfavorable y, en tercer lugar, por la interacción de ambos. Este hecho es característico de una sociedad con instituciones extractivas, en donde a las élites y a los políticos poco les interesa el cambio o el pogreso social, y lo único que desean es mantenerse en el poder. En la Tabla 6, se abordan los efectos municipales, pues en el sexto modelo se incluyen únicamente los efectos directos de las variables municipales.

Tabla 6. Resultados de los modelos a nivel de escuela y de municipio

\begin{tabular}{|c|c|c|c|}
\hline Modelo & 6 & 7 & 8 \\
\hline \multirow[t]{2}{*}{ Género } & & $3,185^{* * *}$ & $3,185^{* * *}$ \\
\hline & & $(0,0304)$ & $(0,0304)$ \\
\hline \multirow[t]{2}{*}{ INSE } & & $0,621^{* * *}$ & $0,655^{* * *}$ \\
\hline & & $(0,031)$ & $(0,0318)$ \\
\hline \multirow[t]{2}{*}{ INSEC } & & $1,983^{* * *}$ & $1,927^{* * *}$ \\
\hline & & $(0,0523)$ & $(0,0543)$ \\
\hline \multirow[t]{2}{*}{ INSEX } & & $0,119^{* * *}$ & $0,119^{* * *}$ \\
\hline & & $(0,0092)$ & $(0,0092)$ \\
\hline \multirow[t]{2}{*}{ Tipo-INSE } & & $0,338^{* * *}$ & $0,341^{* * *}$ \\
\hline & & $(0,0361)$ & $(0,0361)$ \\
\hline \multirow[t]{2}{*}{ Tipo-INSEC } & & $-0,780^{* * *}$ & $-0,787^{* * *}$ \\
\hline & & $(0,0742)$ & $(0,0742)$ \\
\hline \multirow[t]{2}{*}{ Tipo } & & $2,141^{* * *}$ & $2,141^{* * *}$ \\
\hline & & $(0,108)$ & $(0,108)$ \\
\hline
\end{tabular}


Tabla 6. Continuación

\begin{tabular}{lccc}
\hline Modelo & 6 & 7 & 8 \\
\hline TMI & $-0,150^{* * *}$ & $-0,0514^{* * *}$ & $-0,0515^{* * *}$ \\
& $(0,00912)$ & $(0,00648)$ & $(0,00646)$ \\
Desempeño fiscal & $0,124^{* * *}$ & $-0,0333^{* * *}$ & $-0,0321^{* * *}$ \\
Conflicto - 1901-1917 & $(0,00698)$ & $(0,00541)$ & $(0,00544)$ \\
& $-0,399^{* *}$ & $-0,0709$ & \\
Conflicto - 1918-1931 & $(0,14)$ & $(0,0988)$ & \\
& $-0,563^{* * *}$ & $-0,566^{* * *}$ & $-0,567^{* * *}$ \\
Violencia 1948-1953 & $(0,129)$ & $(0,0895)$ & $(0,0838)$ \\
& $-0,532^{* *}$ & $-0,0286$ & \\
Tasa de homicidios & $(0,174)$ & $(0,122)$ & \\
Conflicto 1931- INSE & $-0,00509^{* *}$ & $-0,00985^{* * *}$ & $-0,0101^{* * *}$ \\
Conflicto 1931- INSEC & $(0,00187)$ & $(0,00132)$ & $(0,00132)$ \\
& & & $-0,141^{* * *}$ \\
Constante & & & $(0,0295)$ \\
$N$ & & & $0,233^{* * *}$ \\
ICC & $38,87^{* * *}$ & $40,10^{* * *}$ & $(0,0601)$ \\
& $(0,614)$ & $(0,506)$ & $40,00^{* * *}$ \\
& 405.624 & 405.591 & $40,509)$ \\
& 0,263 & 0,065 & 0,065 \\
\hline
\end{tabular}

Nota: errores estándar entre paréntesis. ${ }^{*} \mathrm{p}<0,1,{ }^{* *} \mathrm{p}<0,05,{ }^{* * *} \mathrm{p}<0,01$

Fuente: elaboración propia con datos del Icfes y el CEDE.

En el sexto modelo se aprecia que todas las variables tienen los signos esperados; sin embargo, al incluir las variables a nivel de hogar y de colegio, algunas de las variables asociadas al conflicto dejan de ser significativas y el indice de desempeño fiscal cambia de signo. Es posible pensar que lo anterior se debe a que no todas las externalidades derivadas de la interacción social deben ser positivas y que esta, al ser la más lejana al estudiante, capte los efectos negativos.

En el octavo modelo se omiten las variables no significativas del séptimo modelo y se consideran interacciones entre algunas variables del nivel municipal y de la escuela y el hogar. Se encuentra que en los municipios donde tuvo 
presencia el conflicto, el nivel socioeconómico del hogar tiene un menor impacto que en los municipios donde no hubo. Por otro lado, el INSEC se ve potenciado en esos municipios. La proporción de varianza explicada por los colegios se mantuvo prácticamente inalterada.

Respecto a los conflictos sufridos en el siglo pasado, resulta interesante que aún poseen un efecto sobre el logro educativo, y podría indicar que en esos municipios aún no se ha logrado crear o fortalecer las instituciones. $\mathrm{Al}$ examinar estas interacciones, se observa que, en aquellas zonas con instituciones extractivas, el capital social, captado por la interacción con el INSEC, posee un efecto positivo, lo cual es coherente, pues en estos lugares el éxito de las personas está predeterminado por la cuna: quienes pertenecen a la elite se rodean de la elite y adquieren mejor educación que el resto de los habitantes. Este efecto se compensa con una reducción en el efecto, derivado de las condiciones del hogar.

\section{Conclusiones}

El estudio cuantifica la importancia de los diferentes contextos que están involucrados en el proceso educativo. En las primeras aproximaciones se encuentra que las escuelas llegan a ser responsables de un $30 \%$ de las diferencias en los resultados individuales, mientras los municipios explican un $5 \%$ de la variabilidad en el rendimiento académico. Además, se verifica la alta importancia que tienen los factores asociados al hogar reunidos en el índice socioeconómico.

El estudio valida la importancia del contexto del hogar y del colegio sobre el desempeño académico. Un aumento en el INSE indica que los padres seleccionan los colegios con mejores condiciones socioeconómicas y, de esta manera, se da lugar a fuertes diferencias en el desempeño académico entre los estudiantes en desventaja socioeconómica respecto a los más aventajados. Además, se observa que los estudiantes con ventajas socioeconómicas asisten, en promedio, a los colegios privados.

En síntesis, aunque el contexto municipal es relevante, sus efectos directos son limitados; sin embargo, las interacciones entre este contexto y las variables 
a nivel de hogar y de colegio resultaron significativas al explicar el rendimiento académico. También se encontraron efectos persistentes en el tiempo: algunos de los conflictos ocurridos en el siglo pasado aún tienen efectos sobre la educación, lo que puede indicar que en dichos municipios todavía no se han formado instituciones sólidas. Las variables más contemporáneas como la TMI y la tasa de homicidios también tienen efectos sobre el rendimiento académico.

En el caso del efecto colegio, la importancia de este contexto fue superior al nivel municipal. Se encontró que dicho efecto estaba altamente correlacionado con la calidad de los pares, lo que evidencia la existencia de segregación en el sistema educativo, consecuencia de la segregación residencial y de que los colegios con mejores desempeños son aquellos que tienen a los estudiantes con las mejores condiciones socioeconómicas. Estos resultados manifiestan la necesidad de generar políticas que se transmitan al contexto escolar y al mismo tiempo reduzcan la segregación en el sistema educativo.

La interacción entre la variable de conflicto, periodo 1918-1931, y las variables de contexto familiar y escolar permite concluir que en los municipios donde se presentaron eventos violentos durante el periodo evaluado, los hogares, respecto al promedio, presentan bajas condiciones socioeconómicas, lo cual ejerce una influencia negativa sobre el desempeño académico de los estudiantes. Por su parte, el efecto del estatus socioeconómico de la escuela se ve potenciado, lo que muestra que en aquellos municipios los vínculos sociales, los nexos y las características del grupo ejercen un efecto superior a la del resto del país, lo que manifiesta que en estas zonas la desigualdad es más preponderante.

\section{Referencias}

Acemoglu, Daron \& Robinson, James (2012). Por qué fracasan los paises. Bogotá: Deusto.

Banco Mundial (2009). La calidad de la educacion en Colombia: Un análisis y algunas opciones para un programa de politica. Autor: Bogotá. 
Rodríguez y Correa: Impacto del contexto municipal sobre el desempeño académico individual

BÉNABOU, Roland (1996). "Equity and efficiency in human capital investment: the local connection", The Review of Economic Studies, Vol. 63, No. 2, pp. 237-264.

BLAnco, Emilio (2011). "Efectos escolares sobre los aprendizajes en México: una perspectiva centrada en la interacción escuela-entorno", Papeles de Población, Vol. 17, No. 69, pp. 219-56.

Bourdieu, Pierre (1997). Capital cultural, escuela y espacio social. México D.F.: Siglo XXI.

Brunner, José Joaquín (2010). “Lenguaje del hogar, capital cultural y escuela”, Resvista Pensamiento Educativo, Vol. 46-47, 17-44.

Coleman, James; Campbell, Ernest; Hobson, Carol; McPartland, James; Mood, Alexander; Weinfeld, Frederic \& York, Robert (1966). Equality of Educational Opportunity. Washington, D.C.: US Department of Health, Education \& Welfare, Office of Education.

Cunha, Flavio \& Heckman, James (2007). "The technology of skill formation”, NBER Working Paper Series, No. 12840. National Bureau of Economic Research. Recuperado de htttps:/www.nber.org/papers/w12840 (junio 6 de 2016).

Duarte, Jesús; Bos, María Soledad \& Moreno, Martín (2009). Inequidad en los aprendizajes escolares en America Latina. Banco Interamericano de Desarrollo. Recuperado de https://goo.gl/7MRQTj (junio 6 de 2016).

Galvis, Luis Armando \& Meisel, Adolfo (2010). "Persistencia de las desigualdades regionales en Colombia: un analisis espacial", Documentos de trabajo sobre Economía Regional, No. 120. Banco de la República, Colombia.

GärTner, Felipe Jaramillo (2014). Educación en Colombia: un enfoque municipal. Recuperado de de: https://biblioteca.uniandes.edu.co/ visor_de_tesis/web/?SessionID=L1Rlc2lzXzIwMTUxMDEvNTAwNC 5wZGY\%3D (marzo 3 de 2016). 
Gaviria, Alejandro \& Barrientos, Jorge Hugo (2001). "Determiantes de la calidad de la educación en Colombia", Archivos de Economía, No. 159. Departamento Nacional de Planeación, Colombia.

Goldstein, Harvey (2011). Multilevel Statistical Models. Chichester, West Sussex: J ohn Wiley \& Sons.

Hanusher, Eric A. (1996). "School resources and student performance". En Burtless, G. (Ed.), Does Money Matter?: The effect of school resources on student achievement and adult success (pp. 43-73). Washington D.C.: Brookings Institution Press.

Hanushex, Eric A. (1997). "Assessing the effects of school resources on student performance: an update", Educational Evaluation and Policy Analysis, Vol. 19, No. 2, pp. 141-161. Recuperado de http://hanushek. stanford.edu/sites/default/files/publications/Hanushek \%201997\%20 EduEvaPolAna \%2019\%282 \%29.pdf (marzo 3 de 2016).

ICFes (2010). Metodología de construcción del indice de nivel socioeconómico de los estudiantes -INSE- y de la clasificación socioeconómica-CSE- de los colegios. mimeo

ICFes (2013). Análisis de las diferencias de género en el desempeño de estudiantes colombianos en matemáticas y lenguaje. Recuperado de http://www.icfes. gov.co/docman/investigadores-y-estudiantes-de-posgrado/resultadosde-investigaciones/equidad/986-analisis-diferencias-de-genero-en-eldesempeno-de-estudiantes-colombianos-en-matematicas-y-lenguaje (septiembre 10 de 2016).

Manski, Charles F. (1993). "Identification of endogenous social effects: the reflection problem", The Review of Economic Studies, Vol. 60, No. 3, 531-542.

Mina, Alejandro (2004). "Factores asociados al logro educativo a nivel municipal”, Documento CEDE, No. 002708. Universidad de los Andes.

NúŃez, Jairo; Steiner, Roberto; Cadena, Ximena \& Pardo, Renata (2002). "¿Cuáles colegios ofrecen mejor educacion en colombia?", Documentos 
Rodríguez y Correa: Impacto del contexto municipal sobre el desempeño académico individual

CEDE, No. 00397. Universidad de los Andes. Recuperado de https:// ideas.repec.org/p/col/000089/003796.html (septiembre 10 de 2016).

Orejuela, Jaime (2014). “Análisis del desempeño estudiantil en las pruebas de estado para educación media en colombia mediante modelos jerárquicos lineales”, Ingeniería, Vol. 18, No. 2, pp. 54-67. Recuperado de: http://revistas.udistrital.edu.co/ojs/index.php/reving/article/view/5231 (septiembre 20 de 2016).

Raudenbush, Stephen \& Bryk, Anthony (1986). "A hierchical model for studying school effects", Sociology of Education, Vol. 59, No. 1, pp. 1-17.

Robinson, James (2016). “The Misery in Colombia”, Desarrollo y Sociedad, Vol. 76, pp. 9-90.

Rozo, Jacobo A. (2015). "La educación secundaria y sus dos dimensiones, efectos del barrio y del colegio sobre los resultados saber 11", Revista de Economía del Rosario, Vol. 20, No. 1, pp. 33-69. Recuperado de: http://repository.urosario.edu.co/handle/10336/10475 (abril 15 de 2016).

Sánchez, Gonzalo \& Peñaranda, Ricardo (Comp.) (1991). Pasado y presente de la Violencia en Colombia. Bogotá: CEREC.

Sen, Amartya (1999). Desarrollo y libertad. Bogotá: Editorial Planeta.

SoAres, José Franciso \& Alves, María Teresa (2013). "Effects of school municipalities in the quality of basic education", Cadernos de Pesquisa, Vol. 43, No. 149, pp. $492-517$.

StataCorp LP. (2013). Stata Multilevel Mixed-Effects. Reference Manual. Texa: Stata Press.

VIvas, Harvy (2008). Educación, background familiar y calidad de los entornos locales en Colombia. Tesis doctoral, Programa de Doctorat d'Economia Aplicada, Departament d'Economia Aplicada, Universitat Autónoma de Barcelona. Recuperado de: http://ddd.uab.cat/record/37984. 
Willms, J. Douglas (2003). Ten hypotheses about socioeconomic gradients and community differences in children's developmental outcomes. Recuperado de: http://citeseerx.ist.psu.edu/viewdoc/download?doi $=10.1 .1 .625 .463 \&$ rep=rep $1 \&$ type $=$ pdf $($ marzo 20 de 2016). 